\title{
Large pulmonary cavity in COVID-19 cured patient case report
}

\author{
Ying Chen ${ }^{1 \#}$, Wanling Chen ${ }^{1 \#}$, Jiansheng Zhou ${ }^{2}$, Cong Sun ${ }^{3}$, Yujie Lei ${ }^{1}$ \\ ${ }^{1}$ Department of Thoracic Surgery I, The Third Affiliated Hospital of Kunming Medical University (Yunnan Cancer Hospital, Yunnan Cancer \\ Center), Kunming, China; ${ }^{2}$ Infections Department, Jiayu County People's Hospital, Xianning, China; ${ }^{3}$ Department of Respiratory and Critical Care \\ Medicine, The Third People's Hospital of Yunnan Province, Kunming, China \\ \#These authors contributed equally to this work. \\ Correspondence to: Yuejie Lei. Department of Thoracic Surgery I, The Third Affiliated Hospital of Kunming Medical University (Yunnan Cancer \\ Hospital, Yunnan Cancer Center), 510 Kunzhou Road, Kunming 650118, China. Email: 58327105@qq.com; Cong Sun. Department of Respiratory and \\ Critical Care Medicine, The Third People’s Hospital of Yunnan Province, 292 Beijing Road, Kunming 650011, China. Email: scdyy0331@sina.com.
}

\begin{abstract}
Coronavirus Disease 2019 (COVID-19) is a pneumonia caused by severe acute respiratory syndrome coronavirus 2 (SARS-CoV-2). The outbreak began in Wuhan, China, and spread rapidly, with many cases confirmed in multiple countries. Usually, after viral pneumonia were clinical cured, the pulmonary lesions of majority patients will gradually be absorbed to complete dissipation, very few severe patients may retain pulmonary interstitial inflammation and fibrosis. In this case, we described one unique COVID-19 patient, the symptoms were: dry cough, fatigue, poor appetite and subjective fever, moreover, the patient was a non-smoker, had no pulmonary bullous, no history of tuberculosis, and also no hypertension or diabetes. The patient received antiviral therapy, antibacterial therapy, recombinant human interferon$\alpha 2 \mathrm{a}$, vitamin $\mathrm{C}$ and oxygen inhalation. After two weeks of treatment and observation, the patient was clinical cured and discharged. However, two days later, the patient had a sudden chest stuffiness, CT images indicted: his lung didn't heal like others, but developed a large pulmonary cavity in the lower lobe of right lung. In hospital, the patient showed no symptoms of infection for another 14 days, and the pulmonary cavity remain unchanged. This case suggested: it is important to follow convalescent COVID-19 patients, especially their lung CT images, to make sure a fully recovery.
\end{abstract}

Keywords: Coronavirus Disease 2019 (COVID-19); severe acute respiratory syndrome coronavirus 2 (SARS$\mathrm{CoV}-2)$; pulmonary cavity; case report

Submitted Feb 19, 2020. Accepted for publication May 11, 2020.

doi: $10.21037 / \mathrm{apm}-20-452$

View this article at: http://dx.doi.org/10.21037/apm-20-452

\section{Introduction}

On January, 2020, Chinese authorities confirmed that Coronavirus Disease 2019 (COVID-19) is a pneumonia caused by a novel coronavirus: severe acute respiratory syndrome coronavirus 2 (SARS-CoV-2), and epidemiologic data indicate that person-to-person transmission is occurring fast, with increasing cases reported in multiple countries worldwide. Pulmonary lesions caused by virus infection will gradually be absorbed to complete dissipation, very few severe patients may retain pulmonary interstitial inflammation and fibrosis (1-3). SARS-CoV-2 is a new coronavirus, the repaired process of SARS-CoV-2 infected pulmonary lesions is not well known. We report a unique COVID-19 case, which developed a large pulmonary cavity in early recovery stage.

We present the following case in accordance with the CARE reporting checklist (available at http://dx.doi. org/10.21037/apm-20-452).

\section{Case presentation}

\section{Patient}

On January 17, 2020, a 34-year-old man presented to Yujia County People's Hospital, Xianning, Hubei, China. 
with a 4-day history of dry cough, fatigue, poor appetite and subjective fever. The patient was a non-smoker, had no pulmonary bullous, no history of tuberculosis, no hypertension or diabetes. On examination his body temperature was $39.5^{\circ} \mathrm{C}$, oxygen saturation $\left(\mathrm{SpO}_{2}\right)$ was $92 \%$, while the patient was breathing, the breathing rate was 21 beats per minute, and the heart rate was 122 beats per minute. On January 18, patient CT images showed bilateral patchy shadows and ground glass opacity in the lungs, and right lung consolidations (Figure 1A). Considering the local COVID-19 epidemiological risk. the patient was admitted as a probable case of SARS-CoV-2 infection.

\section{Diagnosis and treatment}

On January 18, 2020, oropharyngeal swab specimen of the patient was collected, then confirmed to be SARS-CoV-2 positive by reverse transcription-polymerase chain reaction (RT-PCR) assay at January 23, 2020. Blood microorganism culture was performed for 5 consecutive days, and the results were negative (Table 1).

In the hospital, the patient received antiviral therapy (oseltamivir: $75 \mathrm{mg}$, bid), antibacterial therapy (moxifloxacin: $0.4 \mathrm{~g}$, qd; piperacillin sodium tazobactam: $4.5 \mathrm{~g}, \mathrm{qd})$, recombinant human interferon- $\alpha 2 \mathrm{a}: 300$ million units, vitamin C: $3 \mathrm{~g}$ qd and oxygen inhalation.

After 5-day treatment, the patient's body temperature returned to normal, symptoms like dry cough, fatigue and poor appetite disappeared, and $\mathrm{SpO}_{2}$ remained between $95 \%$ to $99 \%$ without oxygen. CT images on January 31 revealed that the shadow of pulmonary plaques was significantly absorbed (Figure 1B). The RT-PCR tests were negative at January 29 and January 30 respectively, reaching the clinical cured standard, so the patient was discharged for observation at home on January 31.

On February 2, the patient returned to the hospital, and he reported a sudden chest stuffiness. CT images showed that the multiple plaques in both lungs were significantly absorbed compared with the previous images, but in the lower lobe of the right lung, a $6.2 \mathrm{~cm} \times 8.6 \mathrm{~cm}$ cystic cavity change appeared, with a small amount of fluid inside (Figure 1C). The patient was readmitted to the hospital for isolated observation, during the observation, the patient had no special symptoms or discomfort. CT reexamination on February 13 showed that the bilateral patchy ground glass opacity in the lungs and the cystic lumens in the right lower lobe were not significantly changed compared with the previous images. The symptoms and treatment timeline were listed in Figure 2.

Due to the local COVID-19 epidemic situation, the medical resources needed to be used for incoming patients, since this patient had no symptoms of infection for another 14 days, he was discharged for home observation. During that time, the patient didn't report any discomfort or adverse events, and didn't return to hospital.

\section{Clinical laboratory results}

Clinical laboratory results showed that alanine transaminase, aspartate aminotransferase, C-reactive protein, creatine kinase, neutrophils and creatine kinase all increased, on the contrary lymphocytes decreased in the early infection stage. Five days later, except for higher platelets, all other indicators returned to normal (Table 2).

\section{Discussion}

COVID-19 is a pulmonary infection disease caused by SARS-CoV-2. This virus can reproduce in bronchiole mucosa or alveolar epithelial cells, resulting in mixed injury of pulmonary parenchyma and interstitial tissue of alveoli, interlobular septa, and peri-acinar microvascular network $(4,5)$. For most cured viral pneumonia, the lung lesions will gradually be absorbed and dissipated, but for COVID-19, patients' image change in convalescence period need further observation.

In 2003, SARS another aggressive coronavirus also caused viral pneumonia pandemic in China. Notably, for SARS patients in later stage of recovery, pulmonary secondary infections caused by mold, tuberculosis and other bacteria were very common, especially for severe patients who used antibiotics, glucocorticoids and ventilator for a long time $(6,7)$. After clinical treatment of viral pneumonia, the lung lesions are gradually absorbed and dissipated, for a few severe cases, fibrosis can be left in the lung. However, literature reported that 11 SARS patients developed pulmonary cavity after bacterial infection in the later recovery stage, with an average time of 41 days $(8,9)$. All 11 patients were treated with long-term, high-dose antibiotics, antiviral drugs and glucocorticoids, and received non-invasive positive pressure mechanical ventilation in the critical stage, after these treatments, the pulmonary cavity disappeared within 1-3 months. However, among the 11 patients, 5 of them died.

In this case, the patient was healthy and had no smoking history. During the course of the disease, no bacteria were 


\section{A}
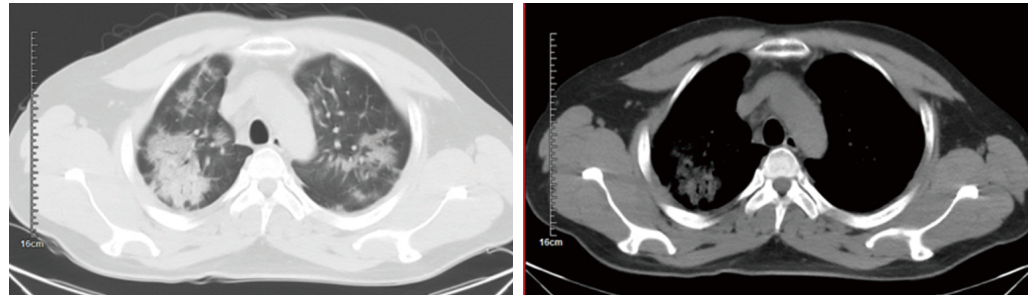

B
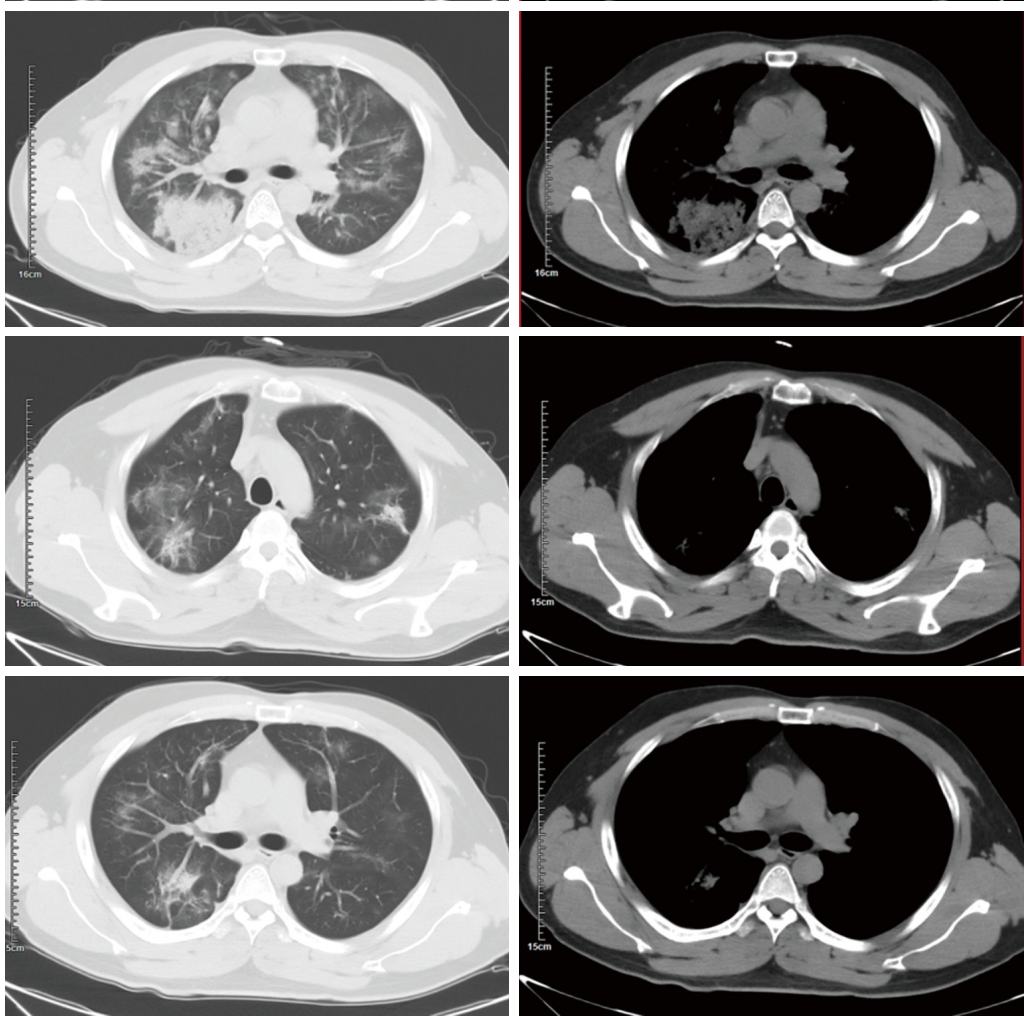

C
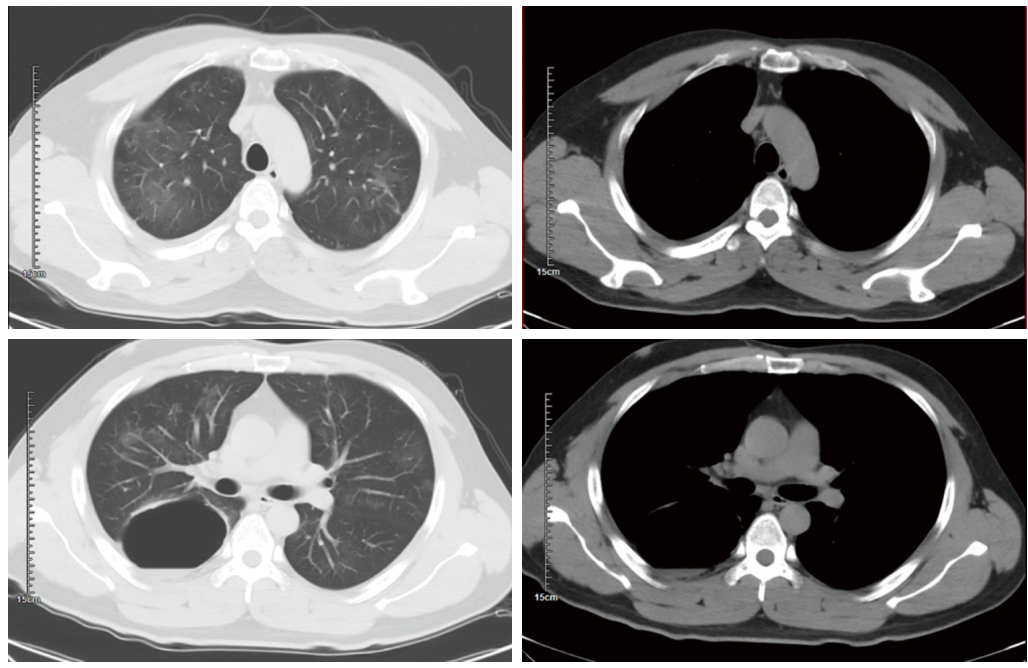

Figure 1 Chest CT images of the patient. (A) Chest CT images on Jan 18, 2020 (Illness Day 7) revealed bilateral GGO and right lung consolidations. (B) Chest CT images on Jan 31, 2020 (Illness Day 20, Discharge Day) showed GGOs in both lungs were decreased obviously, right lung lesions were remarkably resolved. (C) Chest CT scans on Feb 2, 2020 (Illness Day 22, Readmission) showed GGO infections in both lungs remarkably resolved, right lung had a big cavity with liquid inside (GGO, ground-glass opacity). 
Table 1 Clinical laboratory results

\begin{tabular}{|c|c|c|c|c|c|c|c|}
\hline & & Illness day 6 & Illness day 9 & Illness day 13 & Illness day 15 & Illness day 17 & Illness day 22 \\
\hline Lymphocyte count $\left(\times 10^{9} / \mathrm{L}\right)$ & $0.8-4$ & 0.57 & 0.89 & 1.73 & 1.86 & 1.79 & 1.69 \\
\hline Neutrophil ratio (\%) & $50-70$ & 89.6 & 73.1 & 63.9 & 63 & 5.18 & 56.5 \\
\hline Platelet count $\left(\times 10^{9} / \mathrm{L}\right)$ & 85-303 & 239 & 356 & 480 & 483 & 431 & 231 \\
\hline C-reactive protein (mg/L) & $0-10$ & 125.6 & 35.4 & 1.2 & 0.5 & 0.5 & 0.5 \\
\hline Alanine aminotransferase (U/L) & $0-45$ & 57 & 56 & 50 & 40 & 35 & 30 \\
\hline Aspartate aminotransferase (U/L) & $0-45$ & 129 & 62 & 27 & 18 & 21 & 26 \\
\hline Creatine kinase (U/L) & 24-195 & NE & 777 & NE & NE & 136 & NE \\
\hline Creatine kinase isoenzyme (U/L) & $0-25$ & NE & 39 & $\mathrm{NE}$ & NE & 26 & NE \\
\hline
\end{tabular}

*, local LAB; NE, not evaluated.

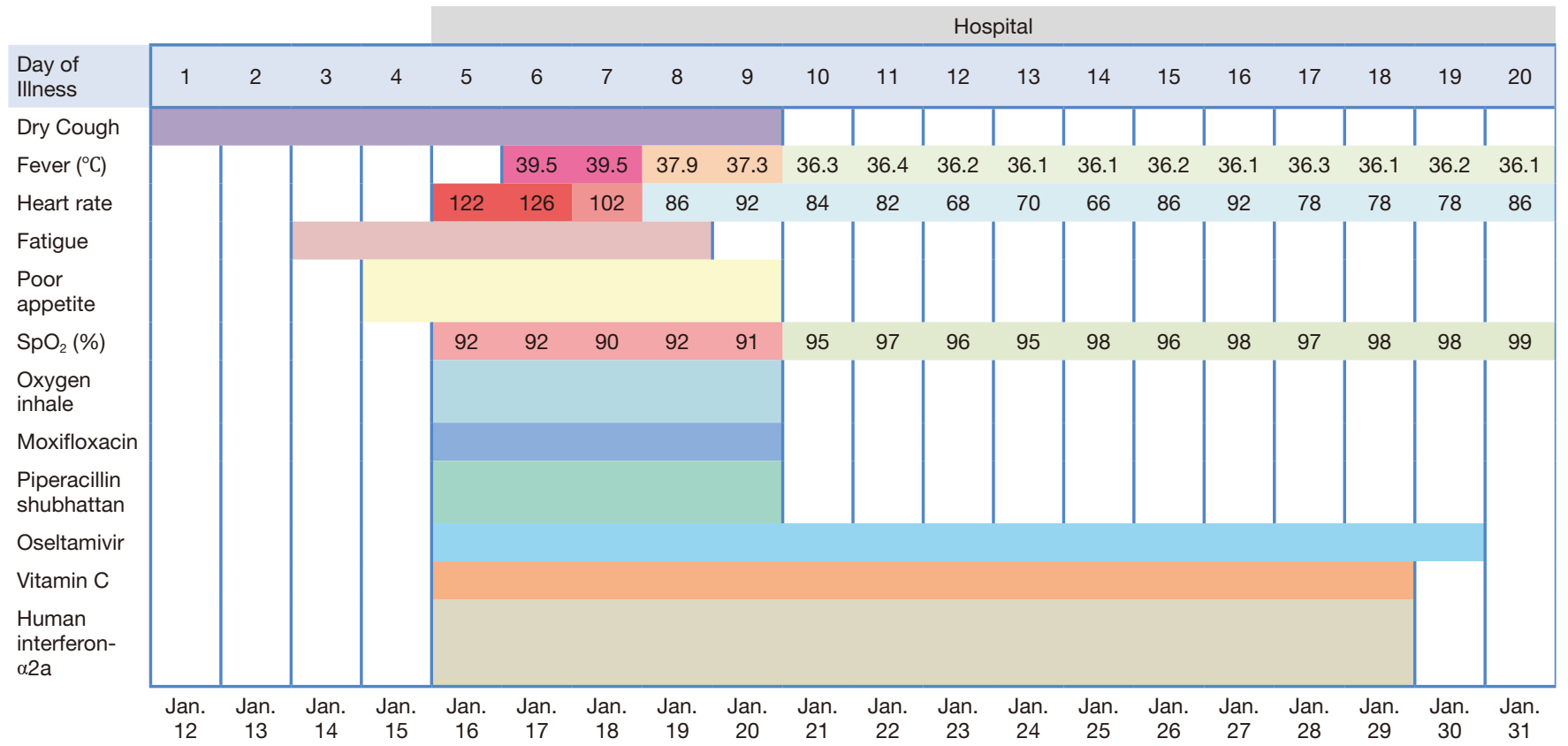

Figure 2 The symptoms and treatment timeline. When the patient presented to the hospital, he had symptoms of dry cough, fatigue, poor appetite and subjective fever. In the hospital, the patient received antiviral therapy (oseltamivir: $75 \mathrm{mg}$, bid), antibacterial therapy (moxifloxacin: $0.4 \mathrm{~g}$, qd; piperacillin sodium tazobactam: $4.5 \mathrm{~g}$, qd), recombinant human interferon- $\alpha 2 \mathrm{a}: 300$ million units, vitamin C: $3 \mathrm{~g}$ qd and oxygen inhalation. 
Table 2 Results of RT-PCR testing for the COVID-2019 and microbial blood culture

\begin{tabular}{|c|c|c|c|c|}
\hline Specimen & IIIness day 7 & Illness day 12 & Illness day 17 & Illness day 19 \\
\hline Nasopharyngeal swab & + & NE & - & - \\
\hline Oropharyngeal swab & + & $\mathrm{NE}$ & - & - \\
\hline \multicolumn{5}{|l|}{ Microbal blood culture } \\
\hline
\end{tabular}

${ }^{*}$, microbal blood culture last 5 days. RT-PCR, reverse transcription-polymerase chain reaction.

detected in the blood microbial culture, and also the results of white-cell count and procalcitonin did not support bacterial infection. In this patient, the large pulmonary cavity appeared on day 22 , additionally, the antibioticsusing time was short and glucocorticoids were not used in the treatment. As a result, the pulmonary cavity of this patient was considered only caused by SARS-CoV-2 infection, possibly leading to a large area of alveolar and surrounding pulmonary interstitial destruction and fusion. Since the local hospital lacking fungus detection equipment, no fungus detection was conducted.

When bacterial infections happened after viral pneumonia and forms a cavity in the lung, the bacteria may adhere to the necrotic fibrous tissue of the cavity wall to form a biofilm. The existence of biofilm can induce resistance to therapeutic drugs as well as body immune, thus significantly prolong the course of the disease and making it difficult for clinical treatment $(10,11)$. Whether SARSCoV-2 can coexist with bacteria in human body as biofilm remains to be studied further.

At present, this is the first pathological report on pulmonary cavity caused by SARS $-\mathrm{CoV}-2$ infection. With the emergence of a large number of clinically cured COVID-19 patients, it is particularly important to followup the convalescent patients, especially their lung CT images, to make sure the fully recovery of the patients. The limitations about this case is that: fungus infection also cause pulmonary diseases, and could possibly form biofilms, if fungus detection was conducted, the data could provide more information on how the large pulmonary cavity was formed.

\section{Acknowledgments}

We would like to thank the nurses and clinical staff who provided treatment and care for the patient; We thank the patient; Special thanks to all the RESCUE team members from the Third Affiliated Hospital of Kunming Medical University (Yunnan Cancer Hospital, Yunnan Cancer Center).

Funding: Yunnan health training project of high-level talents (H-2018025, D-2016041).

\section{Footnote}

Reporting Checklist: The authors have completed the CARE reporting checklist. Available at http://dx.doi.org/10.21037/ apm-20-452

Conflicts of Interest: All authors have completed the ICMJE uniform disclosure form (available at http://dx.doi. org/10.21037/apm-20-452). The authors have no conflicts of interest to declare.

Ethical Statement: The authors are accountable for all aspects of the work in ensuring that questions related to the accuracy or integrity of any part of the work are appropriately investigated and resolved. All procedures performed in studies involving human participants were in accordance with the ethical standards of the institutional and/or national research committee(s) and with the Helsinki Declaration (as revised in 2013). Written informed consent was obtained from the patient for publication of this manuscript and any accompanying images.

Open Access Statement: This is an Open Access article distributed in accordance with the Creative Commons Attribution-NonCommercial-NoDerivs 4.0 International License (CC BY-NC-ND 4.0), which permits the noncommercial replication and distribution of the article with the strict proviso that no changes or edits are made and the original work is properly cited (including links to both the formal publication through the relevant DOI and the license). See: https://creativecommons.org/licenses/by-nc-nd/4.0/. 


\section{References}

1. Wong KT, Antonio GE, Hui DS, et al. Severe acute respiratory syndrome: thin-section computed tomography features, temporal changes, and clinicoradiologic correlation during the convalescent period. J Comput Assist Tomogr 2004;28:790-5.

2. Wu X, Dong D, Ma D. Thin-Section Computed Tomography Manifestations During Convalescence and Long-Term Follow-Up of Patients with Severe Acute Respiratory Syndrome (SARS). Med Sci Monit 2016;22:2793-9.

3. Chung M, Bernheim A, Mei X, et al. CT Imaging Features of 2019 Novel Coronavirus (2019-nCoV). Radiology 2020;295:202-7.

4. Liu K, Fang YY, Deng Y, et al. Clinical characteristics of novel coronavirus cases in tertiary hospitals in Hubei Province. Chin Med J (Engl) 2020;133:1025-31.

5. Pan Y, Guan H, Zhou S, et al. Initial CT findings and temporal changes in patients with the novel coronavirus pneumonia (2019-nCoV): a study of 63 patients in Wuhan,
China. Eur Radiol 2020;30:3306-9.

6. Chiang CH, Shih JF, Su WJ, et al. Eight-month prospective study of 14 patients with hospital-acquired severe acute respiratory syndrome. Mayo Clin Proc 2004;79:1372-9.

7. Leung CW, Chiu WK. Clinical picture, diagnosis, treatment and outcome of severe acute respiratory syndrome (SARS) in children. Paediatr Respir Rev 2004;5:275-88.

8. Yin J, Li K, Liu S, et al. Radiology of Thoracic Complications in SARS. Journal of Capital Medical University 2003;24:401-4.

9. Yuan C, Zhao D, Wang W, et al. Imaging manifestations of the cavitation in pulmonary parenchyma of SARS. Chinese Journal of Radiolgy 2004;038:466-9.

10. Simões $M$. Antimicrobial strategies effective against infectious bacterial biofilms. Curr Med Chem 2011;18:2129-45.

11. Esteban J, García-Coca M. Mycobacterium Biofilms. Front Microbiol 2018;8:2651.
Cite this article as: Chen Y, Chen W, Zhou J, Sun C, Lei Y. Large pulmonary cavity in COVID-19 cured patient case report. Ann Palliat Med 2021;10(5):5786-5791. doi: 10.21037/ apm-20-452 